\title{
Étude numérique de l'interaction laser matière en régime pico et nanoseconde
}

\author{
J.D. Parisse, W. Marine* et M. Sentis** \\ IUSTI, UMR 6595 du CNRS, Université de Provence, Technopôle de Château Gombert, \\ 5 rue Enrico Fermi, 13453 Marseille cedex 13, France \\ * GPEC, UMR 6631 du CNRS, Faculté des Sciences de Luminy, Case 918, 13288 Marseille \\ cedex 09, France \\ ** IRPHE, UMR 6594 du CNRS, Faculté des Sciences de Luminy, Case 918, 13288 Marseille \\ cedex 09, France
}

\begin{abstract}
Résumé : $L$ 'étude de l'interaction laser matière présente des intérêts multiples tant sur le plan théorique que sur le plan technique. D'un point de vue théorique, elle permet de mieux décrire les propriétés des plasmas hors équilibres. Il est donc essentiel de bien comprendre tous les processus intervenant lors de cette interaction. La modélisation numérique permet de prendre en compte la brièveté des phénomènes étudiés. Cette modélisation est basée sur une approche monodimensionnelle instationnaire qui comprend, l'équation de diffusion des paires électrons trous, l'équation de la chaleur et enfin l'équation de conservation de l'énergie électronique. Le système d'équations obtenv est résolu à l'aide d'un schéma aux différences finies. Le code de calcul est en bon accord avec des résultats numériques déjà existants sur des cas tests picosecondes. La différence entre les longueurs d'ondes UV et IR a été mise en évidence dans le cas des impulsions nanosecondes.
\end{abstract}

\section{INTRODUCTION}

L'étude de l'interaction laser matière présente des intérêts multiples tant sur le plan théorique que sur le plan technique. D'un point de vue théorique, elle permet de mieux décrire les propriétés des plasmas hors équilibre.

Il est donc essentiel de bien comprendre tous les processus intervenant lors de cette interaction. La modélisation numérique permet de prendre en compte la brièveté des phénomènes étudiés

Cette modélisation, basée sur une approche monodimensionnelle instationnaire, comprend l'équation de diffusion des paires électrons trous, l'équation de la chaleur et enfin l'équation de conservation de l'énergie électronique. Le système d'équations obtenu est résolu à l'aide d'un schéma aux différences finies.

Le code de calcul est en bon accord avec des résultats numériques déjà existants sur des cas tests picosecondes, les seuls disponibles dans la littérature [1,2]. Il a été utilisé pour simuler des impulsions nanosecondes. Ce qui a permis une comparaison entre les longueurs d'ondes UV et IR dans le cas d'impulsions nanosecondes.

\section{RESULTATS}

Les résultats présentés dans cet article concernent uniquement les impulsions nanosecondes. La cible utilisée pour nos simulations est en Silicium cristallin. L'impulsion laser est, dans tous les cas, de forme gaussienne et de largeur à mi-hauteur de $15 \mathrm{~ns}$.

La figure 1 présente une comparaison entre une longueur d'onde $\mathrm{IR}(530 \mathrm{~nm})$ et une longueur d'onde UV $(193 \mathrm{~nm})$. L'énergie incidente du laser est dans les deux cas de $150 \mathrm{~J} / \mathrm{cm}^{2}$. Sur ce graphe on peut noter qu'il existe un équilibre entre la température et la température électronique (i.e. : $T=T e$ ) dans le cas $530 \mathrm{~nm}$. En revanche il y a un fort déséquilibre entre la température et la température électronique dans le cas $193 \mathrm{~nm}$. Ceci montre que l'hypothèse, couramment employée, d'interaction laser-matière purement thermique n'est pas valable dans le cas des longueurs d'ondes UV. Il est à noter aussi que dans le cas d'une impulsion laser IR, la température atteinte est plus importante que dans le cas UV. Ceci vient $\mathrm{du}$ fait que le coefficient de réflectivité du silicium cristallin est d'environ $10 \%$ plus grand pour la longueur d'onde $193 \mathrm{~nm}$ que pour la longueur d'onde $530 \mathrm{~nm}$. 


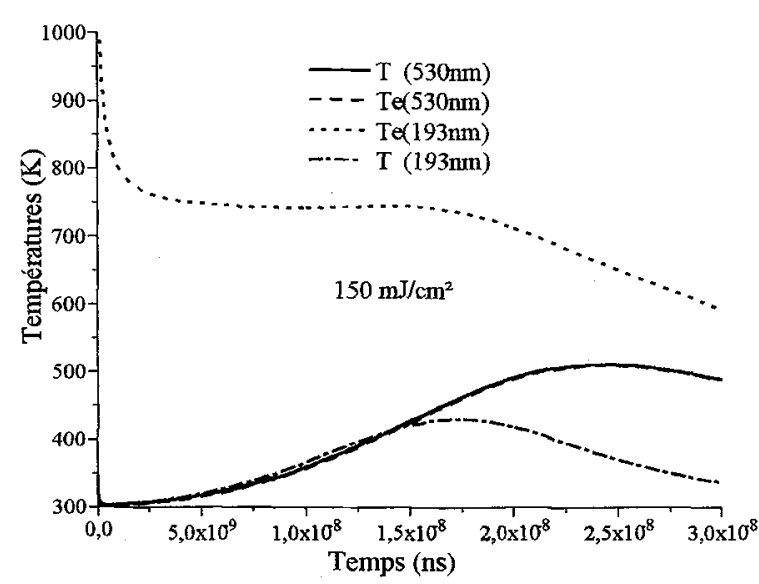

Figure 1 : Evolution de la température et de la température électronique à la surface de la cible de silicium.

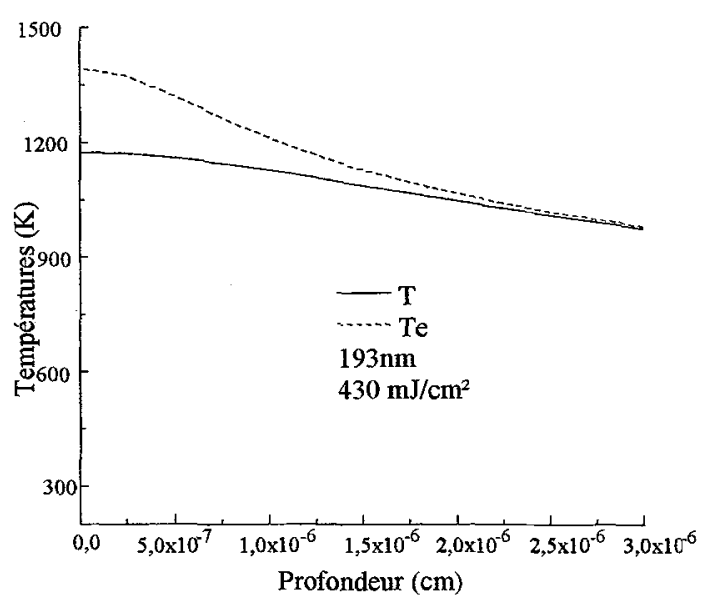

Figure 2 : Répartition de la température et de la température électronique au bout de $15 \mathrm{~ns}$.

Les répartitions de la température et de la température électronique sont représentées sur la figure 2 au maximum de l'impulsion laser $(15 \mathrm{~ns})$. Cette figure montre que le déséquilibre électronique n'existe pas seulement en surface mais aussi en profondeur dans la cible de silicium. Il est à noter que l'écart entre la température et température électronique n'existe que sur environ $30 \mathrm{~nm}$. Cette distance correspond à la distance moyenne de diffusion des porteurs libres les conditions d'applications. On peut en conclure que la zone de déséquilibre électronique correspond à celle de diffusion des porteurs libres.

\section{CONCLUSION}

La modélisation numérique a permis de montrer l'importance du déséquilibre électronique lors d'une interaction laser-matière pour une longueur d'onde UV. Ce travail s'inscrit dans un projet plus vaste visant à développer un code de calcul permettant de simuler la formation d'un plasma induit par ablation laser UV

\section{Références}

1. S.S. Mao, Xlanglei Mao, Jong H. Yoo, Ralph Greif, and Richard E.Russo «Thermionic electron emission from narrow band-gap semiconductors under picosecond laser excitation », Journal of Applied Physics 1998, Volume 3 (206-211)

2. H.M. van Driel « Kinetics of high-density plasmas generated in Si by 1,06 and $0.53 \mu \mathrm{m}$ picosecond laser pulses », Physical Review B 1987, Volume 35, Number 15 (8166-8176) 